\title{
Status of the AFP project in the ATLAS experiment
}

\author{
Marek Taševský, on behalf of the ATLAS Collaboration
}

\author{
Institute of Physics, Academy of Sciences of the Czech republic, Na Slovance 2, 18221 Prague, Czech republic
}

\begin{abstract}
Status of the AFP project in the ATLAS experiment is summarized. The AFP system is composed of a tracker to detect intact, diffractively scattered protons, and of a time-of-flight detector serving to suppress background from pile-up interactions. The whole system, located around $210 \mathrm{~m}$ from the main ATLAS detector, is placed in Roman Pots which move detectors from and to the incident proton beams. A typical distance of the closest approach of the tracker to these beams is 2-3 mm. The main physics motivation lies in measuring diffractive processes in runs with not a very high amount of pile-up.
\end{abstract}

Keywords: diffractive processes; rapidity gap; tagged protons; Roman Pots; fast timing detectors PACS: $13.85 . \mathrm{Hd}$

\section{INTRODUCTION}

A sizable part of the total proton-proton cross section (about 30\%) comes from the diffractive processes defined by one or both incident protons emerging from the interaction point (IP) undetected in the main detector and by an activity in the central region. In addition, the scattered leading protons are accompanied by large rapidity gaps, i.e. large regions of the ATLAS detector [1] devoid of any signal above the noise thresholds of respective subdetectors. The detection of the leading protons and/or of the large rapidity gaps is a powerful tool to select genuine diffractive events. At high instantaneous luminosities the gaps are spoiled by particles from additional pile-up events. The ATLAS detector is equipped with a forward proton subdetector ALFA placed at $240 \mathrm{~m}$ from the IP. Based on scintillator fibres and designed to measure elastic processes and the total pp cross section [2], it is restricted to operate at very low values of $\mu$, numbers of pile-up interactions per bunch crossing. The ATLAS project concentrating on studying diffractive processes, AFP (ATLAS Forward Proton) aims to install forward proton detectors at a distance of $210 \mathrm{~m}$ from the IP. Compared to ALFA detectors put in vertical Roman Pots (RP), the AFP detectors would be put in horizontal RPs where the acceptance to diffractive processes is maximized [3]. In 2011 the AFP project has been approved as a part of the Letter of Intent for the phase-I Upgrade [4] by the ATLAS collaboration which has then been endorsed by the LHC Committee.

\section{PHYSICS MOTIVATION}

The physics motivation for the AFP project is based on investigating diffractive and photon-induced processes. By tagging one leading forward proton, one can study Single Diffractive (SD) and photoproduction processes, by tagging both leading forward protons, one can study Double Pomeron Exchange (DPE) or Central exclusive Production (CEP) processes. Prospects for the diffractive program in the ATLAS experiment were outlined e.g. in [5].

\section{Diffractive processes}

The SD and DPE processes serve to provide information about the low- $x$ structure of the proton and the diffractive parton density functions (dPDFs). Inclusive jet and heavy quark production are mainly sensitive to the gluon component of the dPDFs, while vector boson production is sensitive to quarks. The kinematic region expands that explored at HERA and Tevatron, with values of $\beta$ (the fractional momentum of the struck parton in the diffractive exchange) as low as $10^{-4}$ and of $Q^{2}$ up to $10^{4} \mathrm{GeV}^{2}$. The measurement of the $\mathrm{W}$ boson production asymmetry [6] or the $\gamma+$ jet production [7], both in DPE processes allows one to constrain the quark content of dPDFs and to probe different models of diffraction and to distinguish e.g. between Soft Color Interaction models and Resolved Pomeron models.

The CEP process (see $[8,9,10]$ and references therein) is defined as $\mathrm{pp} \rightarrow \mathrm{p} \oplus \mathrm{X} \oplus \mathrm{p}$ and all of the energy lost by the protons during the interaction ( $\mathrm{a}$ few percent) is used in the production of the central system, $\mathrm{X}$. The final state 
therefore consists of a centrally produced system (e.g. di-jet, heavy particle or Higgs boson), two very forward protons and no other activity. The ' $\oplus$ ' sign denotes the rapidity gaps. In DPE process, the central system contains remnants from the diffractive exchange in addition to the hard subprocess. Among others, the CEP is attractive by the fact that from precise measurements of proton momentum losses, $\xi_{1}$ and $\xi_{2}$, the mass of the central system can be measured much more precisely than from the dijet mass measured in the calorimeters, by the so-called missing mass method, $M^{2}=\xi_{1} \xi_{2} s$ ( $s$ is the squared centre-of-mass energy). The CEP of dijets has already been observed at Tevatron [11] and studied in great detail for the AFP physics program [12]. Results based on PYTHIA 8 generator level and the LHC proton transport program MAD-X [13] show that for events selected by the AFP acceptance and a presence of two jets with transverse momentum above $20 \mathrm{GeV}$, assuming a conservative timing resolution of $30 \mathrm{ps}$ and requiring only one-vertex (to suppress the pile-up background), a purity well above $60 \%$ can be achieved for $\mu \sim 3$. The event yield would be optimal for $\mu$ around 1 but still manageable up to 5 .

\section{Photon-induced processes}

As the LHC beams act also as a source of high energy photons, a rich program of photon-photon and photon-proton processes can be pursued in an unexplored region of phase-space. Although the final state topology is similar to that of CEP, the photon-induced processes may be separated from CEP because their average proton transverse momentum is significantly lower. A process thoroughly studied in the AFP acceptance is the W-pair production in $\gamma \gamma$ interactions where both bosons decay leptonically. The studied quartic anomalous coupling $\gamma \gamma \mathrm{WW}$ appears predominantly at high two-photon masses which, together with the exclusivity, makes this process easy to observe and extract from background. Studies based on a fast simulation of ATLAS and AFP detectors at 220 and $420 \mathrm{~m}$ and including numerous sources of background [14] show sensitivities being $10^{4}$ times better than those measured by the OPAL experiment [15] and $10^{2}$ times better than using the central detector only [16]. With a detailed simulation of ATLAS, with AFP stations at $210 \mathrm{~m}$ only and with high pile-up, similar results are obtained [4].

\section{AFP DESIGN}

One arm of the AFP detector consists of two stations (AFP1 at $204 \mathrm{~m}$ and AFP2 at $212 \mathrm{~m}$ ) of Roman pots that allow the detectors to be in a retracted position during the injection of protons into the LHC, and remotely positioned close to the beam after stable beam collisions are established. AFP1 will contain a 6-layer silicon tracker and AFP2 will contain both the tracking detector identical to the AFP1 station and the ToF detector. The aim of this setup, mirrored by an identical arm placed on the opposite side of the ATLAS IP, is to measure the momentum, angle, and time-of-flight of protons emerging intact from the pp interactions. The latter allows us to reconstruct with a $\sim 2 \mathrm{~mm}$ precision the pp interaction vertex thereby efficiently reject overlapping background events in which the two tagged protons did not originate from the same vertex as the signal.

\section{Roman Pots}

Roman Pots as movable devices hosting tracking sensors are already in use at LHC (Totem experiment [17] and ALFA subdetector), so the AFP collaboration relies on an ample experience with operating and designing RPs. In order to minimize multiple scattering and secondary particle emission, proton will enter RPs through a thin window whose thickness must be as low as possible - currently $250 \mu \mathrm{m}$ is foreseen for a window made of stainless steel. In order to maximize the physics acceptance, it is also necessary to minimize the distance between the detectors and the beam including the thickness of window. The range of motion of RPs can vary from 15 to $35 \mathrm{~mm}$.

\section{Silicon tracker}

The tracker when combined with the LHC dipole and quadrupole magnets, forms a powerful momentum spectrometer. To reconstruct the mass of the central system produced in ATLAS, it is necessary to measure precisely the distance from the beam, while the transverse momentum needs a precise measurement of the angle of the proton tracks relative 
to the beam. With a typical LHC beam size at $210 \mathrm{~m}$ of $\sigma \sim 100 \mu \mathrm{m}$, the window surface can theoretically safely approach the beam to $15 \sigma \approx 1.5 \mathrm{~mm}$. The thickness of the thin window is approximately $250 \mu \mathrm{m}$ and the dead region of the sensors ranges between 10 and $200 \mu \mathrm{m}$ giving the closest distance of about $2 \mathrm{~mm}$. This corresponds roughly to the range $0.015<\xi<0.15$ and consequently to the range roughly $0.25<M<1.5 \mathrm{TeV}$. The key requirements for the silicon tracking system at $210 \mathrm{~m}$ are: i) spatial resolution of $\sim 10(30) \mu \mathrm{m}$ per detector station in $x(y)$-direction, ii) angular resolution for a pair of detectors of about $1 \mu \mathrm{rad}$, iii) high efficiency over an area of $20 \mathrm{~mm}$ x $20 \mathrm{~mm}$, iv) minimal dead space at the edge of the sensors, v) sufficient radiation hardness. The basic building unit of the AFP tracker is a module consisting of a sensor array and on-sensor read-out chip, passive components and electrical services for low voltage, high voltage and signal routing. The module will be mounted on a mechanical support with embedded cooling and other necessary services. The AFP has chosen the 3D Silicon type of sensor and the FE-I4 read-out chip, both having been developed for other upgrade tracking projects in ATLAS. AFP will use 3D double-sided sensors developed by CNM (Barcelona) and FBK (Trento) producers for the IBL project [18]. One sensor is composed of an array of $336 \times 80$ pixels with a pixel size $(x, y)=(50,250) \mu \mathrm{m}$. The 3D sensors have several advantages over the planar technology: they need lower bias voltage, less cooling and are more radiation hard. The FE-I4 is very well suited to the AFP requirements: the granularity of cells provides a sufficient spatial resolution, the chip is radiation hard enough (up to a dose of $3 \mathrm{MGy}$ ), and its size is sufficiently large to serve just one module. This significantly simplifies the design of the tracker. Given all these features, the AFP tracking system is able to sustain a several-year operation at nominal instantaneous luminosities (of up to $10^{34} \mathrm{~cm}^{-2} \mathrm{~s}^{-1}$ ). The more challenging issue is a non-uniform irradiation. Beam tests carried out in 2013 with CNM and FBK sensor prototypes used very non-uniform irradiation profiles. Results presented in [19] show that the overal hit reconstruction efficiency is above $98 \%$ and that bias voltages needed to keep this excellent efficiency in the irradiated regions are of the order of only $100 \mathrm{~V}$. The baseline option for the cooling is the vortex based dry air cooling system going to cool the read-out electronics to temperatures around $-15^{\circ} \mathrm{C}$.
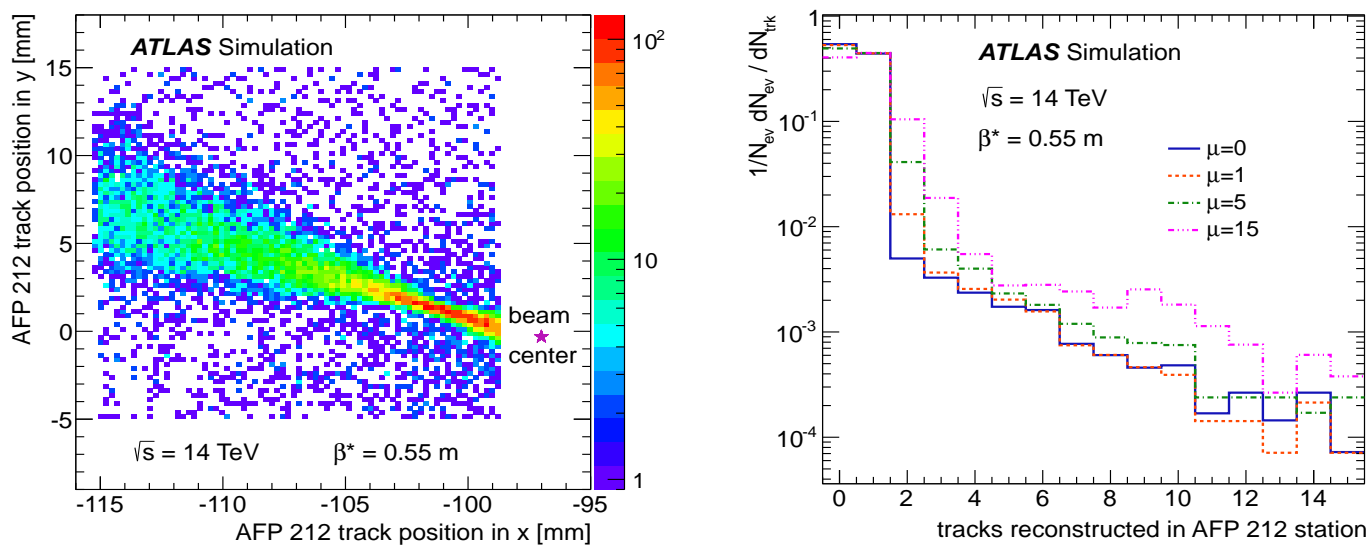

FIGURE 1. Results of the detailed simulation of the AFP system using SD events generated by PYTHIA 8 for pp interaction at $\sqrt{s}=14 \mathrm{TeV}$, in the LHC beam optics $\beta^{*}=0.55 \mathrm{~m}$, and with pile-up events superimposed. A closer look at the outer station of the Silicon detector is given, showers are included and no matching between tracks from the inner and outer station is applied. Left: $(x, y)$-distribution of tracks calculated in the ATLAS coordinate system. Average number of pile-up events per bunch crossing $\mu=1$. Right: Reconstructed track multiplicities for different pile-up scenarios $\mu=0,1,5$ and 15 .

A big progress has been achived in detailed simulation of the whole AFP system. Track reconstruction methods and inefficiencies caused by showers ocurring on the passage of protons from the IP up to the back-end of the ToF detectors are under study. Some results for the outer station of the tracker are shown in Fig. 1. Events were generated by PYTHIA 8 without any cut on the proton kinematics causing about a half of protons in the sample not reaching the AFP acceptance $(0.015<\xi<0.15)$ which results in populating the bin with zero reconstructed tracks. In both plots we see the effect of showers and additional pile-up interactions. Both can be significantly reduced by applying cuts on track $x$ - and $y$-slopes and matching tracks between the inner and outer stations. 
A fast timing system that can precisely measure the time difference between outgoing scattered protons is a key component of the AFP detector to suppress backgrounds coming from pile-up interactions during runs with instantaneous luminosities higher than $10^{30} \mathrm{~cm}^{-2} \mathrm{~s}^{-1}$. The ToF detector should be radiation tolerant, with a good acceptance in the region of interest $(2 \mathrm{~cm} \times 2 \mathrm{~cm})$ and enough segmantion to cover multi-proton events. The proposed timing system, named QUARTIC [20], consists of a quartz-based Cerenkov detector coupled to a microchannel plate photomultiplier tube (MCP-PMT). The signal from MCP-PMT is then amplified, sent to a constant-fraction discriminator and digitized by a high-precision TDC chip (HPTDC [21]). The QUARTIC detector consists of an array of $4 \times 5$ fused silica bars with length from 8 to $12 \mathrm{~cm}$ and oriented at the average Cerenkov angle with respect to the incident proton. To fit the array in RP, two bars connected at $90^{\circ}$ with a $45^{\circ}$ aluminized elbow are added (so called LQ bar design). The performance of the QUARTIC system has been studied at several beam tests during last years at CERN and FNAL, see e.g. [20]. In 2014 beam tests served to study the final LQ bas design, photoelectron yield, resolution, cross talk and the PMT lifetime. In 2012 a 14 ps resolution has been obtained for the average time from six straight bars using $120 \mathrm{GeV}$ proton beams at FNAL. The CFD signal was read out by a fast oscilloscope but replacing it by HPTDC has only a small impact on the final resolution. Alternative detection techniques are also being developed, e.g. Fast Silicon or Dimanond timing detectors, both read out by the SAMPIC chip [22], creating a fruitfull collaboration between ATLAS, CMS and Totem groups. Results from common beam tests showed a 40 ps resolution of the Fast Silicon detector being read out by the SAMPIC chip, while the internal resolution of the chip was measured to be 4 ps. More details about these developments can be found in [23].

\section{SUMMARY AND OUTLOOK}

Several beam tests showed a good progress in the tracking and ToF detector development, including alternative options for the latter. A detailed physics program has been developed. Results on the detailed simulation of the whole AFP system, of the alignment, and of background conditions using data taken by ALFA (not reported here) are promising. The AFP project is currently under evaluation and approval process in the ATLAS collaboration. A most likely scenario for the LHC run II is using the AFP system in special runs with a negligible effect of pile-up, while running at high luminosities is considered as a future option. An instalation of the ToF system at early stages of the AFP operation would be profittable for gaining experience and faster adaptation. A milestone is expected from the beam tests carried out at CERN in November 2014 whose goal is an integrated read-out of the whole system (tracking and timing).

\section{REFERENCES}

1. ATLAS Collaboration, J. Inst. 3 (2008) S08003.

2. ATLAS Collab., arXiv:1408.5778 [hep-ex].

3. M. Trzebinski, arXiv:1408.1836 [physics.acc-ph].

4. ATLAS Collaboration, Letter of Intent of the Phase-I Upgrade of the ATLAS Experiment, CERN-LHCC-2011-012.

5. M. Tasevsky, Nucl. Phys. Proc. Suppl. 179-180 (2008) 187, ATL-PHYS-CONF-2008-019.

6. K. Golec-Biernat et al., Phys. Rev. D84 (2011) 114006.

7. C. Marquet et al., Phys. Rev. D88 (2013) 7, 074029.

8. FP420 R\&D Collaboration, J. Inst. 4 (2009) T10001.

9. V. A. Khoze, A. D. Martin and M. G. Ryskin, Eur. Phys. J. C23 (2002) 311.

10. S. Heinemeyer et al., Eur. Phys. J. C53 (2008) 231.

11. CDF Collaboration, arXiv:0712.0604 [hep-ex].

12. M. Trzebinski, CERN-THESIS-2013-166.

13. F. Schmidt, "MAD-X User's Guide", CERN 2005.

14. C. Royon and M. Saimpert, Int. J. Mod. Phys. A29 (2014) 1446016.

15. OPAL Collaboration, Phys. Rev. D70 (2004) 032005.

16. P. J. Bell, Eur. Phys. J. C64 (2009) 25.

17. Totem Collaboration, J. Inst. 3 (2008) S08007.

18. ATLAS IBL Collaboration, J. Inst. 7 P11010 (2012).

19. J. Lange et al., Proceedings of workshop Pixel 2014; S. Grinstein et al., Nucl. Inst. Meth. in Phys. Res. Sect. A, 730 (2013) 28.

20. A. Brandt, talk at workshop DIRC 2011; J. Pinfold, Acta Phys. Pol. Proc. Suppl. Vol. 4 (2011).

21. M. Mota and J. Christiansen, IEEE journal of solid state circuits, Vol. 34 (1999) 1360.

22. E .Delagnes et al., Nucl. Sci IEEE Trans. 54 (2007) 1735; E. Delagnes, Patent WO 2008/050177, EP2076963.

23. C. Royon and N. Cartiglia, Int. J. Mode. Phys. A29 (2014) 1446017. 